\title{
Correspondence
}

\section{Critical appraisal of the Schizophrenia Commission's report}

I have read with interest the recent report published by the Schizophrenia Commission, ${ }^{1}$ as well as its depictions in the media. I felt that some of the deficits highlighted and suggestions made were reasonable, insightful and helpful. However, some of the recommendations were utopian, unrealistic and a bit simplistic, especially so in this era of austerity. I am not sure whether the Commission considered many of the vicissitudes faced by healthcare professionals when dealing with patients who are actively psychotic, and lack insight and capacity. If these were considered, they did not appear to be promulgated efficiently; this, I think, has been negatively interpreted by the media and has led to undue criticism.

From reading many blogs and internet forums, one can see that there is denigration and sometimes even disdain towards the psychiatric profession by those who perhaps do not have a grasp of the intricacies of the system or of mental illness. Releasing a report without a balanced explanation of these factors might exacerbate this.

Many of the recommendations were helpful. In particular, I envisage that extending general practitioners' training in mental illness could relieve the burden on secondary services, and I was impressed by the novel suggestion of focusing on mental health education in the school curriculum; it could reduce ignorance and stigma and hopefully instil confidence within this generation to seek help for themselves and for their loved ones in the future if necessary.

Other recommendations, although sound in theory, are potentially improbable when the National Health Service (NHS) is struggling with an egregious $E 20$ bn budget cut, with mental health carrying a disproportionate amount of this load (the report itself highlights that mental illness accounts for $23 \%$ of NHS disease burden, yet receives only $13 \%$ of funding - a statistic that has been voraciously quoted in related newspaper articles). These proposals include increasing access to psychological therapies for individuals with schizophrenia, a radical overhaul of acute care and finding gainful employment for them.

There was a large emphasis on the fact that 'increasing number of people are having compulsory treatment ... levels of coercion have increased year on year and are up by $5 \%$ in the last year', as well the Commission's clear view that 'shared decision making on medication choices is essential to improve outcomes'. These are basic principles of good practice that any decent psychiatrist should know and strive towards; I do not think their mention sheds any new light on the issue. On the contrary, for the very significant cohort of patients with schizophrenia who are insightless and lack capacity (whom the report failed to comment on), in reality the choices they make might not be in their best interests. What about the patient with chronic schizophrenia who refuses all medication, but is rescued from florid psychosis, severe deterioration and a salubrious itinerant lifestyle thanks to their regular depot given under the provisions of their Community Treatment Order? I do not believe that 'shared decision making on medication choices is essential' to help this person. Of course, it is obvious to any psychiatrist that the report is not referring to this type of patient. But I fear that the media, the general public, and the disgruntled patient are not cognizant of this.

Similarly, 'recovery houses' are exulted as a cost-effective setting for patients compared with in-patient wards. I absolutely agree that this is the apposite choice for many patients whose psychotic symptoms have assuaged, and who have improved since admission and are close to discharge and independence. But what about patients who are not? The report fails to mention that patients in the community have much less supervision and support, and reduced access to doctors; there is a much higher chance of relapse owing to non-adherence to medication and boundaries. We all know of patients who repeatedly fail because they are discharged into the community prematurely. This is a puissant financial burden on the NHS. We can all make the distinction between the patients who are well and who are not. But can the media and the public? I think there is a danger that this issue can be misinterpreted and the archaic stereotype of the megalomaniacal, over-controlling psychiatrist could be reinforced.

The report particularly denigrates high secure services, stating that 'people stay too long in very expensive and often unsuitable provision'. It does not offer an alternative route for those in high secure settings, such as being released into the community (partially treated and at risk of committing violence) or being transferred to prison (partially treated and at risk of deteriorating). Far from tackling this highly intricate area, the report relays that 'we think preoccupation with risk (important though this is) has forced psychiatrists to rely increasingly on compulsion'. The report seems to be written in basic terms, for accessibility to the lay man. Despite this, there is no mention of the Mental Health Act, its stringent and specific criteria for detention, and the regular mandatory independent legal provisions (such as hospital managers' hearings and mental health review tribunals), which ensure that continued detention is appropriate; I believe these pertinent omissions could be misleading to the lay man, leading him to believe that there are no safeguards against unlawful and immoral detention.

In addition, I take issue with the document's statement that 'there appears to be no clear criteria for different categories of secure provision and a lack of effective care pathways for people in the secure system'; I work in a medium secure forensic psychiatric unit. We have stringent guidelines about levels of security; each case is discussed at length by a coterie of experts on an admissions panel using a standardised, detailed, structured professional judgement instrument (the Dundrum Quartet), where several specific criteria are considered.

Another area of concern for me was the media interpretation of the report. The document did highlight some strengths in provisions (although it did so sparingly), such as the 'positive developments in growth in service user movement, initiatives like crisis resolution teams and early 
intervention for psychosis services, exercise programmes', and also stated 'We are aware of some excellent serviceswhere anyone would be confident to recommend a friend or family member be treated'. I have seen several media depictions of the report, including in the Guardian, BBC News website and the Independent; they were all strongly pessimistic as regards the healthcare system and condemning of the treatment of schizophrenia.

I appreciate that there are shortcomings and areas that need to be highlighted. As mentioned, the report has achieved this in some areas, to some extent. However, many of the deficits promulgated are insolvable without extra resources; focusing on them without offering resources or solutions creates unrealistic and unattainable expectations and sets services up to fail. Additionally, the report propagates many shortcomings (especially as regards patient involvement and community services) without confronting the uncomfortable truth: that many patients with schizophrenia, especially if actively psychotic, or without insight, motivation or capacity, need high levels of care and guidance, and yes, sometimes it is against their will. Without clearly explaining these factors to the cynical general public, and the sensationalist media, there is a danger that frustrations and disappointments towards the illness might be projected on (and thereby disempowering) those that try their best to treat it, under unfavourable conditions and inviolable boundaries.

The report demurs at the stigma of schizophrenia and encourages battle against it, and rightly so. But what does it do for the stigma of psychiatrists?

1 Schizophrenia Commission. The Abandoned IIIness: A Report by the Schizophrenia Commission. Rethink Mental Illness, 2012.

Sohom Das, Specialty Registrar, John Howard Centre, East London NHS Foundation Trust, London, UK, email: sohom.das@eastlondon.nhs.uk

doi: $10.1192 / \mathrm{pb} .37 .2 .72$

\section{Can old age psychiatry attract trainees to psychiatry?}

As an old age psychiatrist with a liaison commitment, I agree with Solomon \& Ranjith. ${ }^{1}$ If the Royal College of Psychiatrists and training programme directors want to address the recruitment issue that troubles psychiatry, we need to be more proactive about how we attract people into the specialty.

For many years, general adult psychiatry has been the focus of government and health board investment which resulted in super-specialisation. Old age, on the other hand, has not had this prioritisation, with the result that we have remained generalists, with multifaceted skills. For this very reason old age psychiatry is appealing: by keeping control of our service, we keep our autonomy and thus our job satisfaction. Retaining a mode of working which provides a varied working week appeals to would-be general practitioners (GPs), who appreciate the continuity provided by community working with the luxury of getting to know their patients.

Working with older people requires the use of to-date medical knowledge on a daily basis, particularly general medicine and neurology. Old age liaison in particular requires medical knowledge gained at university and in postgraduate jobs. Being based in general hospital it demands not only the synthesis of medical and psychiatric knowledge, but the ability to communicate at many levels, thus making it particularly appealing to those potential psychiatrists for whom being a 'real doctor' is important.

The problems encountered by Dr Dudleston ${ }^{2}$ are symptomatic of New Ways of Working gone awry. It is concerning that a scheme conceived by the College and the Department of Health as a way of reducing workplace stress for psychiatrists has been misinterpreted and misapplied by managers, resulting in disempowerment of the role of the consultant psychiatrist, as well as huge regional variation in how services are provided and how training occurs, both at undergraduate and postgraduate levels. ${ }^{3-5}$

Old age psychiatry is the perfect vehicle for multidisciplinary working. I could not do my job without the support of my community mental health team, a group of autonomous individuals whose skills allow me as the medic in the team to focus on the more complex and medical needs of our patients, whether as in-patients, out-patients or in liaison service. This symbiotic relationship has not evolved from New Ways of Working; rather, it has come about from a realisation that the skills of the medic in the team are better used for diagnosis, treatment, clinical decision-making, risk management and service development.

There is something to be said for drawing people into the specialty with the familiar and comfortable. Once secure in their choice, they can explore the diverse and fascinating subspecialties that psychiatry can offer. It is easier to defend a choice when you know that at many levels psychiatry is not that much different to other branches of medicine. We have something to learn from our colleagues in palliative care and oncology who do not doubt the worth of the jobs they do because of lack of a 'cure', and neither should we.

Below are the views of two psychiatric trainees who started out with very different career paths.

From academic pathology to old age psychiatry - I note with interest the recent discussion regarding the decline in the number of psychiatric trainee applicants. As someone who was until shortly before application time 'sold' on another specialty, I feel a personal perspective on why I changed my mind may be of interest. Following an intercalated degree in anatomy and an honours paper discussing histopathological techniques, I was sure I was destined for a career in pathology. I pursued several modules in pathology, a taster week and a placement in my FY2 year. I also undertook a placement in psychiatry (old age) in FY2, which proved to be a turning point. As I was doing an academic foundation programme, I was of course interested in what fields of research were being pursued at that time. Although more is being revealed about organic causes of psychiatric conditions as the research methods become more sophisticated, there remains much to be discovered. This makes it a very exciting time to be part of psychiatry. Working in an old age psychiatry placement highlighted both the organic component of psychiatric conditions and the requirement for research into these, in particular conditions pertinent to an aging population. This placement also changed my mind regarding old age psychiatry as it came across as a more medical specialty owing to patients having a number of comorbidities which require medical treatment, or indeed having an acute confusional state that is the result of a medical insult. Foundation year placements may aid to show that 